\title{
Analog least mean square loops for self-interference cancellation in in-band full-duplex systems
}

\author{
Anh Tuyen Le ${ }^{1}$, Le Chung Tran² ${ }^{2}$ Xiaojing Huang ${ }^{1}$ and Jay Guo ${ }^{1}$ \\ ${ }^{1}$ University of Technology Sydney \\ 2 University of Wollongong \\ Ictran@uow.edu.au
}

\begin{abstract}
Analog Least Mean Square (ALMS) loop is a promising method to cancel self-interference (SI) in in-band full-duplex (IBFD) systems. In this talk, the steady state analyses of the residual SI powers in both analog and digital domains are derived in frequency domain. It is proved that the ALMS loop amplifies the frequency components of the residual SI at the edges of the signal spectrum in the analog domain, but the matched filter in the receiver chain reduces this effect. This results in a significant improvement of the interference suppression ratio in the digital domain before information data detection. The lower bounds of the interference suppression ratio given by the ALMS loop in both analog and digital domains are then addressed. These lower bounds are proved to be joint effects of the loop gain, tap delay, number of taps, and transmitted signal properties. The discovered relationship among these parameters allows the flexibility in choosing appropriate parameters when designing the IBFD systems under given constraints. Finally, the effects of $1 / Q$ imbalance in the ALMS loop and the upper bound of the degradation on SI cancellation performance are then investigated. The degradation is proved to be insignificant even under severe conditions of I/Q imbalance. The upper bound of the degradation provides an essential reference for the system design.
\end{abstract}

Dr Le Chung Tran received the B. Eng. (Telecommunications) degree with the first-class honour and highest distinction from Hanoi University of Transportation and Communications, and the M. Eng. (Telecommunications) degree with the highest distinction from Hanoi University of Science and Technology in Vietnam in 1997 and 2000, respectively. He received Ph.D. in telecommunications engineering from University of Wollongong, Australia, in 2006. From 1997 to 2012, he was a lecturer at Hanoi University of Transportation and Communications, Vietnam. From 2005 to 2006, he was an Associate Research Fellow at Wireless Technologies Laboratory (WTL), University of Wollongong. From 2006 to 2008, he was a Postdoctoral Research Fellow at the University of Luebeck, Germany, under the Humboldt Research Fellowship. Since 2009, he has been with the University of Wollongong, Australia, where he is now a Senior Lecturer. He has achieved numerous awards, including World University Services (WUS) awards (twice), Vietnamese Government's doctoral scholarship, International Postgraduate Research Scholarship (IPRS), prestigious Alexander von Humboldt (AvH) research fellowships (twice), and the Vice-Chancellor's award for Outstanding Contribution to Teaching and Learning (OCTAL). He is the coauthor of 85 publications to date. His research interests include 5G, loT, multiple-input multiple-output (MIMO) systems, ultra-wideband (UWB) communications, software defined radio (SDR), wireless body area networks (WBANs), and signal processing for wireless communications. 